\title{
La universidad ante las posibilidades de los dispo- sitivos móviles en el aprendizaje ubicuo.
}

\author{
Ma Angeles Pascual Sevillano \\ Universidad de Oviedo \\ apascual@uniovi.es
}

\section{Resumen}

El uso de dispositivos móviles de forma aplicada al desarrollo de competencias personales, académicas y profesionales nos sitúa en un marco de actuación universitaria basado en un nuevo paradigma educativo, el de aprendizaje ubicuo. En este artículo se exponen algunos resultados sobre el uso, beneficios, dificultades y competencias adquiridas mediante el uso del libro electrónico en el ámbito universitario español. Las limitaciones se reducen y el acceso al conocimiento salva obstáculos que existían hasta ahora. La tecnología se hace cada vez más accesible y nos acerca la cultura.

Palabras clave: Aprendizaje ubicuo, libro electrónico, ebook, competencias.

The university before the possibilities of the mobile devices in the ubiquitous learning.

\begin{abstract}
The use of mobile devices of form applied to the development of personal, academic and professional competitions places us in a frame of university action based on a new educational paradigm, that of ubiquitous learning. In this article some results are exposed on the use, benefits, difficulties and competitions acquired by means of the use of the electronic book in the university Spanish area. The limitations diminish and the access to the knowledge saves obstacles that existed till now. The technology becomes increasingly accessible and the culture brings us over.
\end{abstract}

Key Words: Ubiquitous learning, electronic book, eBook, competitions.

\section{Referencia normalizada:}

Pascual Sevilla, M. A. (2013) La Universidd ante las posibilidades de los dispositivos móviles en al aprendizaje ubicuo. Historia y Comunicación Social. Vol. 18 Nºspecial Noviembre. Págs. 461-468.

Sumario: 1. Introducción. 2. Metodología de la investigación. 3. Resultados y discusión. 4. Conclusiones. 5. Referencias Bibliográficas.

\section{Introducción}

Los resultados del estudio que presentamos pretenden ser un ámbito de reflexión sobre las aportaciones que los diferentes dispositivos móviles pueden ofrecer al nuevo paradigma educativo del aprendizaje ubicuo. 
La concepción del aprendizaje y donde tiene lugar en el ámbito universitario ha cambiado en los últimos años. Hablar de aprendizaje ubicuo nos sitúa en un contexto caracterizado por el uso de la tecnología y su aplicación en los posibles escenarios de aprendizaje.

Uno de los dispositivos que comienza a entrar con determinación en nuestra sociedad es el libro digital o eBook. Según el último barómetro de la Federación de Gremios de Editores de España (FGEE, 2012), el porcentaje de población española que lee en un libro electrónico ha pasado de un $1,3 \%$ en 2010 a un $6,6 \%$ en $2012 \%$ y según el JIISC National E-books Observatory Project en Inglaterra el $65 \%$ del personal académico y de los estudiantes universitarios afirman utilizar el libro electrónico como apoyo informativo al trabajo y al estudio; En España las ventas de ebooks respresentan el $2,4 \%$ de la facturación del sector, y esta tendencia se repite en el resto de países europeos con la excepción del Reino Unido, donde la venta de libros electrónicos supone el 6\% del total de ventas según los datos de The Publishers Association (PA). Sin embargo en Estados Unidos el mercado del eBook, está madurando a pasos agigantados.

El libro electrónico, también conocido como e-book, eBook, ecolibro o libro electrónico, es una versión electrónica o digital de un libro. Y se pueden utilizar diversos dispositivos pueden ser utilizados como soporte de un libro electrónico como un PC, un PDA, un portátil, una tableta y en general cualquier dispositivo que posea una pantalla y memoria. Sin embargo, los dispositivos específicos para el libro electrónico poseen una apariencia que permite emular la versatilidad del libro de papel tradicional. En su diseño, se buscó movilidad y autonomía (dispositivos móviles con bajo consumo de energía para permitir lecturas prolongadas sin necesidad de recargas), pantallas con dimensiones suficientes para mostrar documentos tradicionales (un A4 o un A5) y alto nivel de contraste incluso a plena luz del día. El tamaño de la "página" en pantalla varía de 13 x $18 \mathrm{~cm}$ a 20 x $28 \mathrm{~cm}$ en dispositivos específicos o prácticamente cualquier tamaño en un PC.

Para navegar por el texto se pueden usar las barras de desplazamientos, las marcas digitales de página, y las capacidades de búsqueda de texto. Estos libros utilizan asimismo la estructura común de cualquier libro tradicional, sin embargo contienen mayor número de prestaciones como el contener música, sonidos o animaciones y en el caso de Internet, posibilita enlaces a otras páginas de libros digitales de la red.

En el mercado podemos encontrar una variedad de tipos de eBooks y formatos de archivos:

- WebBooks: son todos aquellos eBooks que pueden ser leídos o descargados de Internet; sólo requiere una computadora y del software de lectura apropiado.

- PalmBooks: se caracterizan por ser leídos en dispositivos como palm pilots o lectores portátiles.

- Ficheros compilados (.exe) incluyen un visor de navegación. 
La cantidad de formatos existentes solo nos permite en este espacio mencionar sus siglas para que el lector pueda realizar acopio de información: DJVU, doc, ePub, HTML, PDF, RTF, lit, mobi, OEB, oPF, prc, aeh, azw, BBeB,CBR / CBZ,CHM,DTB - DAISY,fb2,lrf,pdb, pml,rb, TCR, TR2 - TR3, WOLF.

Tres palabras parecen esenciales entorno al libro electrónico: almacenamiento, organización y difusión.

La convergencia de tecnologías y la proliferación de nuevos servicios basados en audio y video, permiten que la educación actual esté disponible en todo momento, en cualquier lugar, en cualquier medio social (blog, twitter, faceBook...) y, lo más importante, usando cualquier dispositivo. La ubicuidad se convierte en una condición normal para los nativos digitales. Comúnmente se identifica a la Web 3.0 como Web semántica y a la Web 4.0 como Web ubicua. Se diría que la semántica y la ubicuidad se corresponden una a la otra, y de haber alguna prioridad lógica se lo adjudicaría a la ubicuidad.

Existe una relación directa entre la idea de un aprendizaje ubicuo y la capacidad de los dispositivos móviles de proveer entornos educativos altamente interconectados. Las últimas investigaciones señalan que el aprendizaje en todas partes es equivalente a alguna forma de aprendizaje móvil simple, por ejemplo, mediante entornos a los que puede accederse en diversos contextos y situaciones (Liu \& Hwang, 2009). Para garantizar aprendizajes ubicuos es preciso integrar un conjunto de tecnologías móviles en torno a escenarios convergentes, puesto que esas tecnologías amplían las posibilidades educativas de los entornos virtuales de aprendizaje convencionales.

El aprendizaje móvil es un campo de investigación y práctica educativa en rápida expansión. Sin embargo, todavía existe poco trabajo teórico y conceptual con el que explicar la compleja relación entre las características de la rápida evolución tecnológica, su potencial de la educación y el aprendizaje, así como su integración en la vida cotidiana de los usuarios.

En esta investigación se opta por el marco conceptual de la ecología socio-cultural (Pachler, Bachmair \& Cook, 2010), para investigar sobre estos elementos. De acuerdo con este enfoque, el aprendizaje mediante dispositivos móviles se rige por una relación triangular entre las prácticas culturales, las estructuras sociales y la acción de los estudiantes en el proceso educativo:

(a) Prácticas culturales: Por un lado, los dispositivos móviles son cada vez más utilizados para la interacción social, la comunicación y el intercambio; y también, el aprendizaje es visto como creación de significado culturalmente situado dentro y fuera de las instituciones educativas, al tiempo que los medios de comunicación en la vida cotidiana han alcanzado importancia cultural.

(b) Estructuras: Los estudiantes forman parte de la sociedad individualizada de los riesgos, las nuevas estratificaciones sociales, la individualización de la comunicación de masas móvil y la compleja y prolija infraestructura tecnológica, y hacen que los aprendizajes se rijan por los marcos curriculares de las instituciones educativas con 
enfoques específicos hacia el uso de nuevos espacios culturales como recursos para el aprendizaje.

c) Acción de los estudiantes: De manera creciente, los sujetos se ven inmersos dentro de un nuevo hábitat de aprendizaje en el que sus mundos vitales se configuran como medios y recursos potenciales para el aprendizaje, en el que su experiencia es consignada individualmente en relación con las acciones que es capaz de desarrollar, ya sea en su vida cotidiana o formando parte de planes de estudios reglados (Kress \& Pachler, 2007).

La interrelación de estos tres componentes da lugar a una forma de ecología que se manifiesta en un proceso de transformación educativa y cultural emergente.

Partiendo de las consideraciones señaladas hemos querido analizar en esta investigación, diferentes aspectos de relevancia sobre el uso de estos dispositivos móviles entre los estudiantes universitarios orientados al aprendizaje en su formación. Para ello hemos tenido en cuenta 4 variables independientes: edad, estudios, curso y carrera pues consideramos que eran variables que podían determinar el uso de este dispositivo que analizamos, el libro electrónico.

\section{Metodología de la investigación}

El objetivo del estudio es definir un estándar normativizado de competencias para el uso de dispositivos móviles (Ordenador portátil, Teléfono móvil, PDA, tablet-PC, consolas, eBook, etc.) aplicados en entornos educativos universitarios.

Para la investigación se ha diseñado un cuestionario cuya finalidad fue la de obtener datos cuantitativos/cualitativos sobre los usos de dispositivos móviles por parte de estudiantes de educación superior.

Bajo una etnografía multisituada se pretendió comprender las dinámicas socioculturales que soportan el uso de dispositivos móviles en la población objeto de estudio. En la investigación se aplicó la etnografía como método inductivo, practicado, holístico, transcultural y que tiene por finalidad describir y analizar el substrato cultural de las construcciones sociales.

El cuestionario fue dirigido a los estudiantes de educación superior usuarios de dispositivos móviles en sus prácticas cotidianas. Y la selección de la muestra se llevó a cabo mediante un proceso de segmentación que favoreciera la representatividad de las instituciones de educación superior españolas. En una fase inicial de la investigación se recogieron datos de dos universidades españolas con una muestra válida de 461 estudiantes de edades comprendidas entre los 18 y 26 años de las titulaciones de: Artes y Humanidades (6,1\%), Ciencias (4,8\%), Ciencias Sociales (62\%), Ciencias de la salud (11\%) y Arquitectura e Ingeniería (13\%). Para el análisis de los datos hemos 
recurrido a la técnica de estructuración de documentos textuales por medio de "Minería de textos" (QDA Miner 3.24).

Recurrimos a agrupar los impactos por modalidades o variantes. Esta visión segmentada aconsejó elaborar un prototipo de categorías que nos permitiera diferenciar dentro del macroconcepto aprendizaje, subcategorías explicativas y aclaratorias de la categoría principal, aprendizaje. Y así nos pareció pertinente denominarlas "motivos" que tenían los estudiantes para buscar los aprendizajes. "Actividades" realizadas que complementan los aprendizajes y se internan en el campo de las estrategias. "Beneficios" de conocimiento y procesuales obtenidos y "Lugares de uso". Buscamos siempre los términos clave, identitarios y situacionales que nos podían existencializar el contexto integral obtenido.

\section{Resultados y discusión}

De los 461 cuestionarios realizados a los estudiantes sólo el 11,5\% de ellos utilizan el eBook, de los cuales el 16,6\% lo utiliza de forma combinada con otros dispositivos móviles como puede ser el ordenador portátil o el smarth phone. El 58\% de la muestra que utiliza el eBook son hombres y el $42 \%$ son mujeres. Y si tenemos en cuenta la edad de los relatores oscilaba entre los 19 y 26 años, encontramos algunas diferencias en los grupos de edad que usan el eBook en su actividad cotidiana, son los estudiantes entre los 19 y 20 años los que más utilizan estos dispositivos en su aprendizaje.

Una variable importante en el uso de este dispositivo son los estudios que está cursando el estudiante. En este caso son los estudiantes de las carreras de Ciencias Sociales y Jurídicas los que más utilizan este dispositivo y los que menos los de carreras técnicas.

La aplicación de la metodología expuesta permitió seleccionar un diccionario de conceptos agrupado por macrocategorías referenciado a los conceptos de la investigación. Este diccionario de conceptos de componentes y subcomponentes del mapa competencial del eBook contiene un total de 4 macrocategorías: actividades didácticas, dificultades, beneficios y competencias genéricas desarrolladas.

En relación a las actividades didácticas que desarrollan con el libro digital vinculadas al aprendizaje nos encontramos con respuestas que constatamos influyen en la mejora del aprendizaje por los estudiantes, porque lo suelen utilizar en los trayectos de transporte público, en clase para consultar libros de temáticas diferentes que comenta el profesor en las asignaturas o en la propia biblioteca. Es habitual su uso como repositorio para llevar todos los libros y apuntes que necesitan y poder consultarlo cómodamente. Por otra parte pueden tener los libros sin tener que pasar físicamente por la librería sino que con bajarlo de internet (previo pago) es suficiente.

Ellos mismos reconocen que el libro digital les ha facilitado el aprendizaje por su comodidad de acceso, transporte y uso en cualquier momento debido a su tamaño. 
También resulta más económico porque hay muchas ofertas o se lo pasa algún compañero. Menor coste, más libros y se enriquece el vocabulario son sus palabras en relación al eBook.

En relación a la macrocategoría "Dificultades encontradas en el uso del libro digital" se han constatado que no todos los libros que les gustaría tener o necesitan se encuentran aún en este tipo de formatos y que no todos los formatos son compatibles. En cuanto al manejo y capacidad del dispositivo la dependencia de una batería es algo que, en ocasiones, les dificulta, así como la sensibilidad de algunas pantallas táctiles para viajar y su resistencia a pequeños golpes.

En relación a la macrocategoría "Beneficios que se obtienen de utilizar el recurso", constatamos algunas respuestas que ya se habían visto en aportaciones al aprendizaje: el tamaño que permite que se pueda utilizar en cualquier lugar y momento, la variedad de libros que puedes tener simultáneamente, la posibilidad que dan algunos modelos de conexión a internet para poder realizar la compra de forma directa, el precio de los libros que suele ser más económico, el ahorro de papel y tinta, las aplicaciones que tienen, permiten aumentar y disminuir el tamaño de la letra, subrayar o repetir las páginas que interesan, incluso algunos comentan la alta duración de la batería.

En la macrocategoría "Habilidades necesarias para el uso de estos dispositivos" los encuestados señalan que realmente son dispositivos sencillos de manejar, se necesita únicamente algún conocimiento básico de informática y descarga de aplicaciones.

En relación a la macrocategoría "Competencias necesarias para el uso del libro digital" constatamos que la competencias lectora y tecnológica son básicas en su manejo y a ellas añadiríamos las competencias cognitivas básicas para el acceso a la información relevantes, la organización de dicha información y la posterior aplicación del conocimiento adquirido bien de forma compartida o plasmada en actividades de carácter individual.

\section{Conclusiones}

El aprendizaje ubicuo implica que el aprendizaje es una experiencia que no se limita a las instituciones educativas formales sino que se da en muchos lugares, y para los jóvenes especialmente, la movilidad y la portabilidad de este tipo de dispositivos significa que el aprendizaje pasa a ser una actividad que se da en cualquier lugar, en cualquier momento. Y las actividades habría que diseñarlas en relación con lo que sucede en los diferentes contextos, tanto el formal como el no formal. Se está brindando una gran oportunidad de investigación en este entorno de aprendizaje transformado y buscar nuevas formas de aprendizaje, nuevas formas que permitan la colaboración entre los estudiantes en este contexto ubicuo. der.

El desarrollo tecnológico actual permite dar pasos hacia una nueva forma de apren- 
Son muchos los dispositivos que permiten ese aprendizaje y entre ellos se encuentran el formato de libro digital que se muestra incipiente en el contexto universitario. Sin embargo los últimos desarrollos ergonómicos y económicos hacen prever una presencia muy activa en el panorama social y académico en los próximos años debido a sus buenas prestaciones, su reducido tamaño y su capacidad de almacenamiento e interacción con la información. Tal y como acabamos de ver en el análisis de las 461 encuestas los estudiantes han encontrado aportaciones de interés al aprendizaje directo e indirecto con estos dispositivos móviles. Las plataformas virtuales empleadas en la formación han de ser conscientes de estos nuevos dispositivos e incorporar aplicaciones que permitan adecuarse a las competencias requeridas en la universidad del siglo XXI.

\section{Bibliografía}

CARBAJO CASCÓN, F GÓMEZ DÍAZ, R.; ALONSO ARÉVALO, J.; CORDÓN GARCÍA, J.A. (2012). Libros electrónicos y contenidos digitales en la sociedad del conocimiento. Madrid. Pirámide.

DESCRIPTORES DE DUBLÍN (2005). Shared "Dublin" descriptors for the Bachelor's, Master's and Doctoral awards. (Draft 1.31 working document on JQI meeting in Dublin. 2004PC).

FOMBONA CADAVIECO, J. (2008). Lectura de imágenes y contenidos. Competencias para el análisis de la forma y contenidos del audiovisual: Hacia una teoría de la composición. CEP. Madrid.

FOMBONA CADAVIECO, J.; MAMPASO, J. (2010). Influencia de las TICS Audiovisuales en el nuevo perfil de alumnado. EduPsykhé. Revista de Psicología y educación. Vol. 9 (1), pp. 61-83.

KRESS, G. and PACHLER, N. (2007). Thinking about the ' $\mathrm{m}$ ' in m-learning. En N. Pachler (Ed), Mobile learning: towards a research agenda. North Tyneside: The WLE Centre, Institute of Education, 7-32.

LIU, G.; HWANG, G. (2009). A key step to understanding paradigm shifts in e-learning: towards context-aware ubiquitous learning. British Journal of Educational Technology, 40(6), 421-450.

PACHLER, N.; COOK, J.; BACHMAIR, B (2010): Appropriation of mobile cultural resources for learning. International Journal of Mobile and Blended Learning, 2(1).

SEVILLANO GARCÍA, M.L.; VÁZQUEZ CANO, E. (2008). Potencialidad de las tecnologías de la información y comunicación en las labores de gestión educativa. Avances en Supervisión Educativa, (8).

Artículos en publicaciones web

RICOY LORENZO, $\mathrm{M}^{\mathrm{a}}$ Carmen, FELIZ, Tiberio, and COUTO OLIVERA, $\mathrm{M}^{\mathrm{a}}$ Joau (2013). The digital divide among university freshmen. TOJET: The Turkish 
Online Journal of Educational Technology, 12(2), 262-268. En http://www.tojet. net/ [23-8-2013]

JISC national e-books observatory project: Key findings and recommendations Final Report, 2009. En http://observatory.jiscebooks.org/reports/jisc-national-e-books-observatory-project-key-findings-and-recommendations/ [20-72013]

M.E.C.D. Hábitos de lectura y compra de libros en España, 2012. En http://www.editoresmadrid.org/media/43692/h\%C3\%A1bitos\%20lectura $\% 20 \mathrm{a} \% \mathrm{C} 3 \% \mathrm{~B} 1 \mathrm{o} \% 20$ 2012.pdf [20-7-2013]

\section{La autora}

$\mathrm{M}^{\mathrm{a}}$ angeles Pascual Sevillano. Licenciada y Doctora en Filosofía y Ciencias de la Educación. Catedrática de Escuela Universitaria del Departamento de Ciencias de la Educación de la Universidad de Oviedo. Directora del Máster en Formación del Profesorado de Educación Secundaria Obligatoria, Bachillerato y Formación Profesional. Temáticas de investigación y publicación: las Tecnologías de la Información y la Comunicación en la Educación, Formación del Profesorado, Docencia Universitaria, Atención a la Diversidad. Participación y coordinación en Programas de Doctorado en el Departamento de Ciencias de la Educación y en convenio con Universidades de América Latina: Cuba, México. 